\title{
WHY NOT USE THE ENTROPY METHOD FOR WEIGHT ESTIMATION?
}

\author{
Masaaki SHINOHARA, Chikako MIYAKE and Keikichi Ohsawa \\ Department of Mathematical Information Engineering \\ College of Industrial Technology, Nihon University \\ 1-2-1 Izumi-chou, Narashino, Chiba 275-8575, Japan \\ m7sinoha@cit.nihon-u.ac.jp
}

Keywords: additive error, linear scale, entropy method, simulation

Summary: When a decision maker decides each element of a pairwise comparison matrix on the basis of linear scale, the entropy method is expected to produce a weight vector the closest to the true weight vector. On the contrary when a decision maker decides on the basis of exponential scale, the eigenvector method and the geometric mean method are expected to produce weight vectors closer to the true weight vector.

\section{Introduction}

Various methods, such as the eigenvector method (EGV), the geometric mean method (GMM), and the entropy method (ENT) [1], have been proposed to estimate a weight vector from pairwise comparison data. In this paper we will show that when a decision maker decides each element of a pairwise comparison matrix $\mathrm{A}=\left\{a_{i j}\right\}$ on the basis of linear scale with regular intervals, among the eigenvector method, the geometric mean method, and the entropy method, the entropy method produces a weight vector which is statistically the closest to the true weight vector.

\section{Multiplicative Error and Additive Error}

Let $\mathrm{A}=\left\{a_{i j}\right\}$ be a measured $\mathrm{n} \times \mathrm{n}$ pairwise comparison matrix, $\mathrm{W}=\left\{w_{i j}\right\}$ be the consistent $\mathrm{n} \times \mathrm{n}$ pairwise comparison matrix, $x_{0}$ be a true weight vector, and $\mathrm{E}=\left\{e_{i j}\right\}$ be an $\mathrm{n} \times \mathrm{n}$ error matrix associated with $\mathrm{A}$ and $\mathrm{W}$. In general, $\mathrm{A}$ can be expressed as a function of $\mathrm{W}$ and $\mathrm{E}$.

$$
A=f(W, E)
$$

In this paper we will consider two types of error functions, multiplicative error and additive error. In case of multiplicative error with error matrix $M, f(W, M)$ is expressed by Eq.(2), and in case of additive error with error matrix $D, f(W, D)$ is expressed by Eq.(3).

$$
\begin{aligned}
& \mathrm{f}(\mathrm{W}, \mathrm{E})=\mathrm{W} * \mathrm{M} \\
& \mathrm{f}(\mathrm{W}, \mathrm{D})=\mathrm{W}+\mathrm{D}
\end{aligned}
$$

Here, the operation $*$ in Eq.(2) indicates the matrix operation for elementwise product, where $d_{i j}=b_{i j} \times$ $c_{i j}$ for $\mathrm{D}=\mathrm{B}^{*} \mathrm{C}$, and the operation + in Eq.(3) is the ordinary matrix addition, where $d_{i j}=b_{i j}+c_{i j}$ for $\mathrm{D}=\mathrm{B}+\mathrm{C}$. 


\section{Theorems concerning Scale Type and Error Type}

Since we have introduced two types of errors in Sec.2, we will give two theorems which relate an error type and a scale type. In the case of a linear scale as in Fig.1, if $w_{i j}\left(=x_{i} / x_{j}\right)$, true judgment value of a decision maker, is distributed uniformly over the whole scale and the decision maker fixes his or her judgment value to $a_{i j}$, which is one of the nearest discrete values, $d_{i j}\left(=a_{i j}-w_{i j}\right)$, the difference from $a_{i j}$ to $w_{i j}$, distributes uniformly over $[-0.5,+0.5]$. Therefore, following Theorem 1 is obtained.

\section{[ Theorem 1]}

If $w_{i j}=x_{i} / x_{j}$, true (or consistent) judgment value of a Decision Making Unit on the 9-stage linear scale for the $(\mathrm{i}, \mathrm{j})$ th element of a measured pairwise comparison matrix $\mathrm{A}=\left\{a_{i j}\right\}$, is distributed uniformly over $[0.5,9.5], d_{i j}$, the $(\mathrm{i}, \mathrm{j})$ th element of the additive error matrix $\mathrm{D}=\left\{d_{i j}\right\}$, is distributed uniformly over $[-0.5,+0.5]$, where $a_{i j}$ takes a discrete value among $1,2,3,4,5,6,7,8$, and 9 .

Smilarly in the case of an exponential scale as in Fig.2, the same discussion holds if we take logarithms on the exponential scale, and following Theorem 2 is obtained.

\section{[ Theorem 2 ]}

If $w_{i j}=x_{i} / x_{j}$, the true ( or consistent) judgment value of a Decision Making Unit on the 5-stage exponential scale for the $(\mathrm{i}, \mathrm{j})$ th element of a measured pairwise comparison matrix $\mathrm{A}=\left\{a_{i j}\right\}$ is distributed log-uniformly over $\left[2^{-0.5}, 2^{4.5}\right], m_{i j}$, the $(i, j)$ th element of the multiplicative error matrix $\mathrm{M}=\left\{m_{i j}\right\}$, is distributed log-uniformly over $\left[2^{-0.5}, 2^{+0.5}\right]$, where $a_{i j}$ takes a discrete value among 1,2 , 4,8 , and 16 .

\section{Simulation Experiment}

In order to compare the three weight estimation methods, EGV, GMM, and ENT, in its weight estimation accuracy under the two types of error structures, following simulation experiment is carried out.

1. Assume a true weight vector $x_{0}$.

$$
\left(\mathrm{n}=4 \text { and } x_{0}=\left(\frac{1}{10}, \frac{2}{10}, \frac{3}{10}, \frac{4}{10}\right)^{T} \text { in our experiment }\right) \text {. }
$$

2. Make consistent pairwise comparison matrix $\mathrm{W}=\left\{w_{i j}\right\}\left(w_{i j}=x_{i} / x_{j}\right)$ from the true weight vector $x_{0}$.

3. Make a set of perturbed pairwise comparison matrices from the consistent matrix $\mathrm{W}$ by applying additive or multiplicative error to each element of $\mathrm{W}$.

4. Apply the three weight estimation methods, EGV, GMN, and ENT, to each of perturbed matrices. Let $x_{1}, x_{2}$, and $x_{3}$ be estimated weight vector by EGV, GMM, and ENT, respectively.

5. For each sample from the perturbed set, calculate $d_{01}, d_{02}$, and $d_{03}$, the distance from the true weight vector $x_{0}$ to each of estimated weight vector $x_{i}(\mathrm{i}=1,2,3)$.

6. Average the distance $d_{0 i}(\mathrm{i}=1,2,3)$ over the perturbed set. 


\section{Experiment Result}

Tables 1 and 2 show averaged distances $\overline{d_{01}}, \overline{d_{02}}$, and $\overline{d_{03}}$, for the case of additive errors distributed uniformly over $[-0.5,+0.5]$ and the case of multiplicative errors distributed log-uniformly over $\left[\frac{1}{\sqrt{2}}, \sqrt{2}\right]$, respectively.

From Table 1 and other related simulation experiment result, it is shown that under the additive error the entropy method produces a weight vector statistically the closest to the true weight vector (as depicted in Fig.3), and from Table 2 and other related simulation experiment result, it is shown that under the multiplicative error both the eigenvector method and the geometric mean method produce weight vectors which are statistically closer to the true weight vector (as depicted in Fig.4).

Table 1. Average distances under additive errors uniformly distributed over $[-0.5,0.5]$

\begin{tabular}{|c|c|c|c|}
\hline & $\mathrm{n}=4$ & $\mathrm{n}=8$ & $\mathrm{n}=12$ \\
\hline$\overline{d_{01}}$ & 0.100940 & 0.060048 & 0.073549 \\
\hline$\overline{d_{02}}$ & 0.101054 & 0.060453 & 0.073924 \\
\hline$\overline{d_{03}}$ & 0.081136 & 0.038540 & 0.050749 \\
\hline
\end{tabular}

Table 2. Average distances under multiplicative errors log-uniformly distributed over $\left[\frac{1}{\sqrt{2}}, \sqrt{2}\right]$

\begin{tabular}{|c|c|c|c|}
\hline & $\mathrm{n}=4$ & $\mathrm{n}=8$ & $\mathrm{n}=12$ \\
\hline$\overline{d_{01}}$ & 0.007990 & 0.004792 & 0.003394 \\
\hline$\overline{d_{02}}$ & 0.007989 & 0.004791 & 0.003393 \\
\hline$\overline{d_{02}}$ & 0.008179 & 0.005308 & 0.004027 \\
\hline
\end{tabular}

\section{Conclusion}

Together with the simulation experiment result in Sec.5 and the two theorems in Sec.3, it is concluded that when a decision maker decides his or her $a_{i j}$-judgment on widely-used linear scale basis, the entropy method is expected to produce a weight vector which is the closest to the unknown true weight vector. To analyze how the error is distributed under more general conditions is one of the future research problems.

\section{References}

[1] Masaaki Shinohara, ,Entropy AHP and its comparison with conventional AHP's", The Fifth International Symposium on The Analytic Hierarchy Process (ISAHP'99.), pp165-170 (1999.8). 


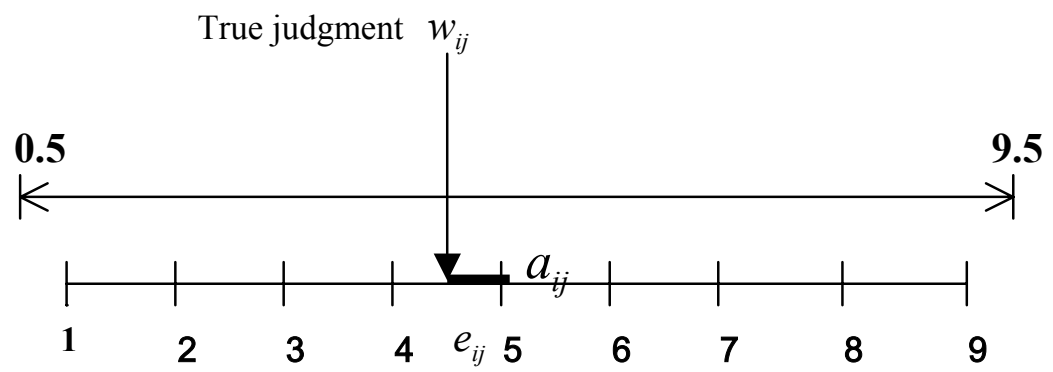

Fig.1 True judgment $w_{i j}$, measured discretized judgment $a_{i j}$, and the error $d_{i j}$ on the 9 -stage linear scale

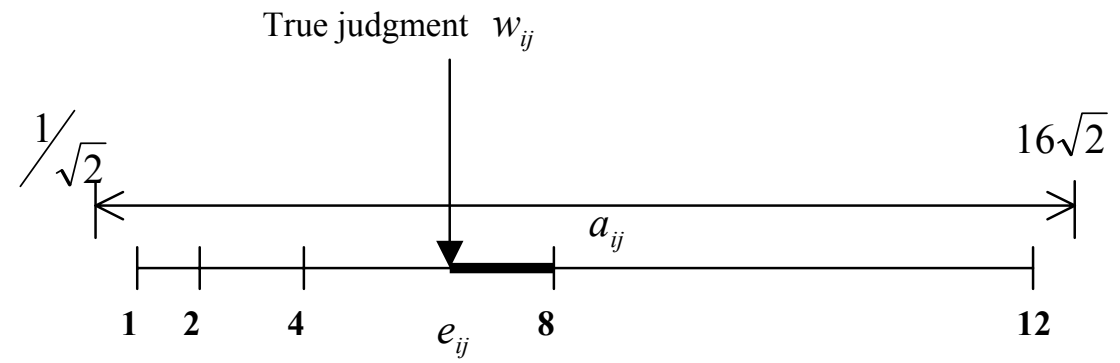

Fig.2 True judgment $w_{i j}$, measured discretized judgment $a_{i j}$, and the error $m_{i j}$ on the 5 -stage exponential scale

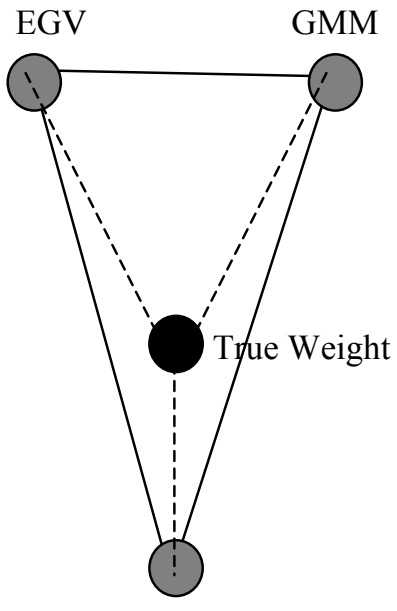

ENT

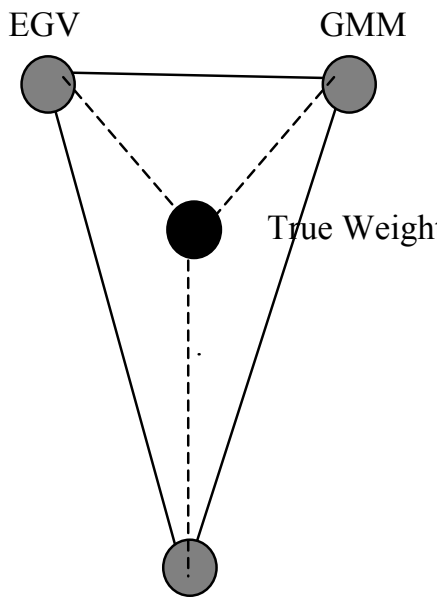

ENT
Fig.3 Schematic diagram for relative position of the four weight vectors under additive error error
Fig.4 Schematic diagram for relative position of the four weight vectors under multiplicative 
